\title{
Factors for predicting early infection after retrograde intrarenal surgery (RIRS) in 1-2 cm renal stones
}

\author{
Huseyin Kazan ${ }^{1}$, Mehmet Caglar Cakici $^{1}$, Ferhat Keser ${ }^{1}$, Meftun Culpan ${ }^{1}$, Ozgur Efiloglu ${ }^{1}$, \\ Asif Yildirim ${ }^{1}$, and Gökhan Atış ${ }^{1}$ \\ ${ }^{1}$ Istanbul Medeniyet University
}

March 23, 2021

\begin{abstract}
Objective: To investigate the factors, especially preoperative urinalysis, predicting postoperative early infection after retrograde intrarenal surgery (RIRS) in 1-2 cm renal stones. Methods: Of the 642 patients who underwent RIRS between September 2013 and July 2019, 289 patients with a total stone size of 1-2 cm were included in the study. Patients were divided into two groups as with and without postoperative urinary tract infection. The demographic data and perioperative findings of all patients were retrospectively reviewed. Sterile urine cultures were obtained in all patients during the preoperative 30-day period and urine analysis values were included in the data. Results: Urinary system infection (UTI) was seen in 20 (6.9\%) of 289 patients. Patient demographics were similar between groups. There was no statistically significant difference between the two groups in terms of stone diameter and stone localization (median diameter 13.5 vs $15, \mathrm{p}=0.285$ ). Patients with postoperative UTI had a higher rate of UTI history ( $55 \%$ vs $20.5 \%, \mathrm{p}=0.000$ ) and longer operative times ( 62.5 vs $60 \mathrm{~min} ., \mathrm{p}=0.008$ ). Rate of pyuria, leukocyte esterase and nitrite positivity were observed more frequently in patients with postoperative UTI. In multivariate analysis, UTI history, prolonged operative time, and nitrite positivity were found to be independent risk factors for postoperative UTI. Conclusion: Nitrite positivity in preoperative urinanalysis, history of UTI and prolonged operation time are the factors that predict the postoperative infection in RIRS for stones between $1-2 \mathrm{~cm}$.
\end{abstract}

Factors for predicting early infection after retrograde intrarenal surgery (RIRS) in 1-2 $\mathrm{cm}$ renal stones

\section{Objective:}

To investigate the factors, especially preoperative urinalysis, predicting postoperative early infection after retrograde intrarenal surgery (RIRS) in 1-2 cm renal stones.

\section{Methods:}

Of the 642 patients who underwent RIRS between September 2013 and July 2019, 289 patients with a total stone size of 1-2 cm were included in the study. Patients were divided into two groups as with and without postoperative urinary tract infection. The demographic data and perioperative findings of all patients were retrospectively reviewed. Sterile urine cultures were obtained in all patients during the preoperative 30-day period and urine analysis values were included in the data.

\section{Results:}

Urinary system infection (UTI) was seen in $20(6.9 \%)$ of 289 patients. Patient demographics were similar between groups. There was no statistically significant difference between the two groups in terms of stone diameter and stone localization (median diameter 13.5 vs $15, \mathrm{p}=0.285$ ). Patients with postoperative UTI had a higher rate of UTI history ( $55 \%$ vs $20.5 \%, \mathrm{p}=0.000$ ) and longer operative times $(62.5$ vs $60 \mathrm{~min} ., \mathrm{p}=0.008)$. Rate of pyuria, leukocyte esterase and nitrite positivity were observed more frequently in patients with 
postoperative UTI. In multivariate analysis, UTI history, prolonged operative time, and nitrite positivity were found to be independent risk factors for postoperative UTI.

\section{Conclusion:}

Nitrite positivity in preoperative urinanalysis, history of UTI and prolonged operation time are the factors that predict the postoperative infection in RIRS for stones between $1-2 \mathrm{~cm}$.

Keywords: Leukocyte esterase, Nitrite, Pyuria, Retrograde Intrarenal Surgery, Urolithiasis

\section{What's known:}

Most common complication of retrograde intrarenal surgery (RIRS) is postoperative urinary tract infection (UTI).

Most established factors of postoperative UTI are antimicrobial resistance, prolonged operation time, preoperative pyuria, and comorbidities as diabetes and obesity.

\section{What's new:}

- This study focused on the predicting role of an overlooked and simple test such as "urinalysis" on postoperative UTI after RIRS.

- Prolonged operation time, UTI history and preoperative nitrit positivity in urinalysis are independent risk factors for postoperative UTI after RIRS.

- Sterile urine culture does not exclude postoperative UTI, nitrit positivity in preoperative urinalysis should be considered.

\section{Introduction:}

Endourological interventions as retrograde intrarenal surgery (RIRS), percutaneous nephrolithotomy (PNL) and shock wave lithotripsy (SWL) are the most common treatment options for kidney stones between 1-2 cm. The European Association of Urology (EAU) and American Urological Association (AUA)/Endourological Society guidelines also suggest these treatment choices $(1,2)$. Retrograde intrarenal surgery exceeds with miniaturized flexible ureteroscopes as a minimally invasive operation with limited complications. Among those complications, RIRS has a complication rate between $9 \%$ and $25 \%$ [2,3]. Urinary tract infection (UTI) is the most frequent complication after RIRS [4,5]. De la Rosette et al. analyzed 11,885 patients who underwent ureteroscopic lithotripsy in the manner of complications, and they reported the most frequent complication as postoperative fever $(1.8 \%)$ and followed by hematuria requiring blood transfusion $(0.2 \%)$. The majority of complications was Clavien grade 1 or 2 (6).

Since postoperative infection is the most common complication, prophylactic empirical antibiotic use has become widespread and its use is now standardized to prevent postoperative UTI. Lo et. al showed that prophylactic empirical antibiotics reduce the rate of bacteriuria, however without clinical impact (7). Because of the empirical use of antibiotics, increasing antimicrobial resistance, and unpredictable patient factors; the rate of postoperative UTI is still high and severe consequences like urosepsis are seen in daily practice.

There have been several studies defining these predictive factors of postoperative UTI. Antimicrobial resistance, prolonged operation time, preoperative pyuria, the stone itself, diabetes, obesity, urinary catheters are some of the most blamed factors for the postoperative UTI $(8,9,10)$. However, there is no consensus on the factors and preventing postoperative infection.

This study aimed to investigate the factors causing urinary tract infection after RIRS to decrease the rate of procedure-related complications. Not only patient and procedure-related factors and also preoperative simple urine characteristics are especially examined.

\section{Material and Methods:}

From September 2013 to July 2019, 642 patients' medical records, who underwent RIRS for proximal ureteral and/or kidney stones, were reviewed retrospectively. 289 patients with a total stone size of 1-2 cm were 
included in the study. Stones between 1-2 cm were selected to stick to the exact and most common treatment indication of RIRS for proximal ureteral and renal stones according to the guidelines. 124 patients whose stones smaller than $10 \mathrm{~mm}, 217$ patients whose stones bigger than $20 \mathrm{~mm}$, and 17 patients with an indwelling urinary catheter or who were doing clean intermittent catheterization were excluded.

Patients were divided into two groups; with and without postoperative urinary tract infection. Demographic data of patients as age, sex, body mass index (BMI), accompanying diabetes mellitus (DM), hypertension (HT), Charlson Comorbidity Index (CCI) and stone diameter, stone location; perioperative characteristics like preoperative hydronephrosis, urinary tract infection (UTI) history, that defined as any symptomatic UTI with positive urine culture, preoperative Double-J stent; operative features like access sheat use, operation time and residual fragments (fragments bigger than $2 \mathrm{~mm}$ ) were retrospectively analyzed.

The postoperative urinary tract infection was defined as incidence within the month of ureteroscopy, fever higher than $38.3{ }^{\circ} \mathrm{C}$ and positive results in urine or blood bacterial culture. Sterile urine cultures were obtained in all patients during the preoperative 30-day period. Urinanalysis values, which were evaluated at the same time with sterile urine culture, as count of leukocytes, presence of pyuria, leukocyte esterase, and nitrite positivity were analyzed. Prophylactic antibiotics were chosen individually for each patient, according to former frequent positive urine cultures; if former urine culture positivity is not present, cefuroxime 1500 mg was chosen for prophylaxis. 7.5 F Storz Flex-X2 was used for flexible URS. A hydrophilic coated ureteral access sheath $(9.5 / 11.5 \mathrm{~F}$ or $12 / 14 \mathrm{~F})$ was preferred.

Statistical analyses were performed using SPSS Statistics ver. 22 software (SPSS, Chicago, IL, USA) using independent samples t-test for comparing the means. When the variables between groups are not normally distributed, mann-whitney u test was preferred. Chi-square test or Fisher's exact test was used for categorical variables. Logistic regression was performed for multivariate analysis and p-value $<0.05$ was considered as statistically significant.

Ethical board approval was granted for this study (2021/0124).

\section{Results:}

Postoperative urinary tract infection (UTI) was seen in 20 (6.9\%) of 289 patients who had RIRS with 1-2 $\mathrm{cm}$ renal and/or proximal ureteral stones. Twenty patients with postoperative UTI were defined as group-1 and 269 patients without postoperative UTI were defined as group-2. The majority of the patients who developed postoperative UTI were female, whereas the rate of male patients was higher in the non-UTI patients ( $55 \%$ vs $39.8 \%, \mathrm{p}=0.181)$. Age, body mass index, accompanying diabetes mellitus, hypertension, Charlson comorbidity index scores were similar between the two groups. Median stone diameters were similar in both groups (median $13.5 \mathrm{~mm}$ vs $15 \mathrm{~mm}, \mathrm{p}=0.285$ ). Patients with postoperative UTI had a higher rate of UTI history ( $55 \%$ vs $20.5 \%, \mathrm{p}=0.000$ ) and longer operative times (median $62.5 \pm 16.6$ minutes vs 60 \pm 19.4 minutes, $\mathrm{p}=0.008$ ). Preoperative hydronephrosis, access sheat use, and residual fragments did not make a difference between two groups. Patients with postoperative UTI had a higher rate of preoperative $\mathrm{Dj}$ stent, but it was also not statistically significant (40\% vs $23.4 \%, \mathrm{p}=0.097$ ) (Table-1).

To predict the postoperative UTI, the features of urinanalysis, which were obtained at the same time with sterile urine cultures, were analyzed. Patients with postoperative UTI had higher leukocyte count in urinanalysis (median 22.5 vs $7.0, \mathrm{p}=0.009)$ and pyuria was more often in this group ( $85 \%$ vs $59.8 \%, \mathrm{p}=0.026)$. Leukocyte esterase positivity was more common in patients with postoperative UTI (90\% vs 63.6\%, p= $0.017)$ and they had higher rates of nitrite positivity (35\% vs $8.6 \%, \mathrm{p}=0.000)$ (Table-2). Besides sensitivity, specificity, positive predictive value, negative predictive value, and the diagnostic odds ratios [Sensitivity/(1sensitivity) / (1-specificity)/specificity] were analyzed for each urine test parameters and their combinations. Nitrite alone has the highest diagnostic odds ratio, whereas a combination of nitrite and leukocyte esterase has the same diagnostic odds ratio. Pyuria and leukocyte esterase have high sensitivity $(85 \%, 90 \%)$ but significantly low specificity rates $(40.1 \%, 36.4 \%)$. Nitrite has low sensitivity ( $35 \%)$ and high specificity $(91.4 \%)$, above all, nitrite has a higher positive predictive value than pyuria and leukocyte esterase $(23.3 \%$ vs $9.5 \%)$ (Table-3). 
In multivariate analysis adjusting for covariates determined by univariate analysis as UTI history, operation time, leukocyte count in urinanalysis, presence of pyuria, leukocyte esterase and nitrite positivity; UTI history, prolonged operative time, and nitrite positivity in preoperative urinanalysis were found to be the independent risk factors for postoperative UTI in RIRS patients with stones between 1-2 cm (Table-4).

The stone analysis couldn't reveal for every patient. In group-1 eight patients (40\%) had stone analysis, most frequent stone type is calcium oxalate (including mixture with calcium phosphate) in 4 patients (50\%); uric acid stones in 1 patient (5\%); struvite stone in 2 patients (\%10) and cystine stone in one patient (\%5). In group-2 eighty-two patients (30.5\%) had stone analysis, the most frequent type is calcium oxalate (including mixture with calcium phosphate) in 63 patients (76.8\%), 12 patients (\%14.6) had uric acid stones and 7 patients $(8.6 \%)$ had cystine stone.

\section{Discussion:}

With the technological advances and miniaturization of the instruments, the most commonly used treatment method in the proximal ureter and kidney stones between 1-2 $\mathrm{cm}$ is retrograde intrarenal surgery. The fact that it is a minimally invasive treatment method allows it to be preferred often, but it can not be said that it has no complications, and the most common complication in daily practice is postoperative urinary tract infection. De Coninck et al. gathered together in their systematic review and reported the incidence of postoperative urinary tract infection after ureteroscopy between $0.2 \%$ and $15 \%$ (12). Up-to-date 14 studies reported the rate of postoperative UTI and various rates might be related to different characterizations of postoperative UTI and also different preoperative preparations and prophylaxis.

In our study $20(6.9 \%)$ of 289 patients had postoperative UTI after RIRS. Our postoperative infection rate seems to be lower than some studies in the literature, and this may have been achieved by obtaining a sterile urine culture in all patients and following a regular and patient-based antibiotic prophylaxis strategy during the operation preparation phase. The fact that it seems to be at a higher rate than some studies may be due to the fact that we are a referral center for stone disease with a high number of complex cases and some unpredictable factors. Which patients faced with postoperative UTIs frequently have been previously investigated in many studies. Some factors, such as female gender, diabetes, hydronephrosis, stone size, presence of a urethral catheter, ureteral

stent, and percutaneous nephrostomy have been expressed mostly in predicting postoperative infection $(10,13,14)$.

Prolonged operation time is a well-described predictor of postoperative UTI after ureteroscopy. There is no consensus in optimal operation time or no time limit, but in order not to keep intrarenal pressure at high levels for a long time, it is aimed at the general approach to keep operation times as short as possible. Southern et al. analyzed the risk factors of postoperative fever and systemic inflammatory response syndrome (SIRS) after 3298 URS procedures. Their postoperative infectious complications rate was $6.9 \%$ and female gender, surgical time, Charlson comorbidity index (CCI) and positive preoperative urine culture were independent predictors. Patients with postoperative infectious complications had an overall longer operative time (57 vs 49 minutes) (15). Moses et al. defined the predictors of postoperative UTI after URS as operation time longer than two hours, pre-stenting, and non-compliance to AUA antibiotic prophylaxis guidelines (16). Kim et al. found operation time as the only independent risk factor for postoperative UTI after URS (82.8 vs 64.5 minutes). They defined 70 minutes as a cut-off value for operative time with the sensitivity of $58.1 \%$ and specificity of $61.7 \%$ (9). In our study, we have also found longer operation time as an independent risk factor for postoperative UTI after RIRS in 1-2 cm ureteral/renal stones (median 62.5 \pm 16.6 vs $60 \pm 19.4 \mathrm{~mm}$; $\mathrm{p}=$ 0.006). Our study differs from other studies, which define similar results, by obtaining sterile preoperative urine culture before the procedure and having similar stone burdens. As we before mentioned, long operative time brings high pressure into the renal pelvis, which leads to systemic absorption of high irrigation volume.

Previous urinary tract infection history is a known risk factor for re-infection. Having such a preoperative history can also help us predict that an UTI may occur in the postoperative period. Mitsuzuka et al. found preoperative acute pyelonephritis as an independent predictor of postoperative UTI after URS (11). In their 
study, it was reported that postoperative complication rates were higher in patients who were treated with antibiotics and then operated. In contrast, we have excluded those patients, and a sterile urine culture was obtained for all patients within 1 month and prepared for the operation. Youssef et al. emphasized that preoperative sepsis leads to higher postoperative UTI and sepsis rates after URS (17). In our study, we have defined the history of UTI as symptomatic UTI over the past year that was accompanied by positive urine culture. It was observed that postoperative infection developed significantly more frequently in the patient group with a history of UTI than in the patient group without ( $55 \%$ vs $20.5 \%, \mathrm{p}=0.000$ ).

In our study, we would especially like to underline that the operation was performed after obtaining a sterile urine culture in the preoperative period in all patients. Obtaining a sterile urine culture is also important, especially considering that these operations lead to intrarenal high pressure. Microbiome studies, which have been frequently mentioned, have also shown that the known sterile genitourinary system is not actually sterile. By the improvements of advanced PCR and 16S rRNA sequencing techniques, we have seen that many organisms colonize the urinary tract (18).

Although obtaining a sterile urine culture is important, especially in stone patients, sterile urine culture can be misleading. A simple urine analysis which we use in routine daily practice, but is overlooked alongside the urine culture. In our study, we have seen that the urine analysis taken at the same time as sterile urine culture may provide us an insight into the development of infection in the postoperative period. The biggest advantage of urine analysis is that it is a simple method that is always obtained. Mitsuzuka et al. observed that preoperative pyuria was associated with postoperative UTI and that postoperative UTI progressed more seriously as the level of pyuria increased. Two patients with severe pyuria progressed to urosepsis (11). Kohada et al. analyzed the risk factors for postoperative UTI after transurethral resection of bladder tumors and they have observed that asymptomatic pyuria was associated with postoperative UTI (19). Chen et al. reported the predictors of postoperative UTI following percutaneous nephrolithotomy. They have characterized the pyuria, nitrite positivity, positive urine culture, and positive stone culture as predictors of postoperative UTI and urosepsis. The coexistence of pyuria and nitrite positivity was found to be the most valuable parameter for predicting postoperative infection and $90 \%$ correlation was found with culture positivity (20). Fan et al. identified the risk factors for developing postoperative uroseptic shock following percutaneous nephrolithotomy. Preoperative urine nitrite, stone size, and postoperative blood leukopenia were the independent risk factors (21). However, in all of these four studies, that investigate preoperative urine analysis, preoperative sterile urine culture was not obtained, contrary to our study. In our study, we have observed that all of the urine analysis parameters play a role in univariate analysis for predicting postoperative UTI. But nitrite positivity is the only independent predictor of postoperative UTI after RIRS (OR: 8.31, $\mathrm{p}=0.000$ ). Additionally, its combination with other urine analysis parameters also did not add additional value in predicting postoperative UTI.

Analyzing urine analysis parameters that may help to predict postoperative infection, despite sterile urine culture is the main strength of our study. It is seen that the studies in the literature, the condition of sterile urine culture was not sought. Our study is the first study done in this aspect. Especially in ureteral/renal stone patients, obtaining preoperative sterile urine culture is very important for us, but sterile urine culture does not guarantee that there will be no infection in the postoperative period. In this study, we aimed to see these factors.

Among the main limitations of our study are retrospective modality, limited stone analysis in all patients, and infection stone rates. Since our clinic is a clinic where complex cases are operated in terms of stone diseases, its applicability to the general can also be seen as a limitation. The limited number of patients, especially in the postoperative infection group, may be counted as a limitation.

\section{Conclusion:}

Nitrite positivity in preoperative urinanalysis, history of UTI, and prolonged operation time is the independent risk factors that may predict the postoperative infection in RIRS for ureteral/renal stones between 1-2 $\mathrm{cm}$. Although preoperative sterile urine culture is important and should be obtained, a simple urine analysis, 
especially nitrite positivity should be also considered. A patient-based approach, considering risk factors, is recommended.

\section{References:}

1. EAU Guidelines. Edn. presented at the EAU Annual Congress Barcelona 2019. ISBN 978-94-92671-042 .

2. Assimos D, Krambeck A, Miller NL, et al (2016) Surgical Management of Stones: American Urological Association/Endourological Society Guideline, PART I. J Urol 196:1153-1160. https://doi.org/10.1016/j.juro.2016.05.090

3. Perez Castro E, Osther PJS, Jinga V, et al (2014) Differences in Ureteroscopic Stone Treatment and Outcomes for Distal, Mid-, Proximal, or Multiple Ureteral Locations: The Clinical Research Office of the Endourological Society Ureteroscopy Global Study. Eur Urol 66:102-109. https://doi.org/10.1016/j.eururo.2014.01.011

4. Geavlete P, Georgescu D, Niţ A G, et al (2006) Complications of 2735 Retrograde Semirigid Ureteroscopy Procedures: A Single-Center Experience. J Endourol 20:179-185. https://doi.org/10.1089/end.2006.20.179

5. Breda A, Ogunyemi O, Leppert JT, Schulam PG (2009) Flexible Ureteroscopy and Laser Lithotripsy for Multiple Unilateral Intrarenal Stones. Eur Urol 55:1190-1197. https://doi.org/10.1016/j.eururo.2008.06.019

6. Kim JW, Lee YJ, Chung J-W, et al (2018) Clinical characteristics of postoperative febrile urinary tract infections after ureteroscopic lithotripsy. Investig Clin Urol 59:335. https://doi.org/10.4111/icu.2018.59.5.335

7. de la Rosette J, Denstedt J, Geavlete P, et al (2014) The Clinical Research Office of the Endourological Society Ureteroscopy Global Study: Indications, Complications, and Outcomes in 11,885 Patients. J Endourol 28:131-139. https://doi.org/10.1089/end.2013.0436

8. Lo C-W, Yang SS-D, Hsieh C-H, Chang S-J (2015) Effectiveness of Prophylactic Antibiotics against Post-Ureteroscopic Lithotripsy Infections: Systematic Review and Meta-Analysis. Surg Infect (Larchmt) 16:415-420. https://doi.org/10.1089/sur.2014.013

9. Kim JW, Lee YJ, Chung J-W, et al (2018) Clinical characteristics of postoperative febrile urinary tract infections after ureteroscopic lithotripsy. Investig Clin Urol 59:335. https://doi.org/10.4111/icu.2018.59.5.335

10. Senocak C, Ozcan C, Sahin T, et al (2018) Risk Factors of Infectious Complications after Flexible Uretero-renoscopy with Laser Lithotripsy. Urol J 15:158-163. https://doi.org/10.22037/uj.v0i0.3967

11. Mitsuzuka K, Nakano O, Takahashi N, Satoh M (2016) Identification of factors associated with postoperative febrile urinary tract infection after ureteroscopy for urinary stones. Urolithiasis 44:257-262. https://doi.org/10.1007/s00240-015-0816-y

12. De Coninck V, Keller EX, Somani B, et al (2019) Complications of ureteroscopy: a complete overview. World J Urol. https://doi.org/10.1007/s00345-019-03012-1

13. Sohn DW, Kim SW, Hong CG, et al (2013) Risk factors of infectious complication after ureteroscopic procedures of the upper urinary tract. J Infect Chemother 19:1102-1108. https://doi.org/10.1007/s10156-013-0632-7

14. Martov A, Gravas S, Etemadian M, et al (2015) Postoperative Infection Rates in Patients with a Negative Baseline Urine Culture Undergoing Ureteroscopic Stone Removal: A Matched Case-Control Analysis on Antibiotic Prophylaxis from the CROES URS Global Study. J Endourol 29:171-180. https://doi.org/10.1089/end.2014.0470

15. Southern JB, Higgins AM, Young AJ, et al (2019) Risk Factors for Postoperative Fever and Systemic Inflammatory Response Syndrome after Ureteroscopy for Stone Disease. J Endourol 33:516-522. https://doi.org/10.1089/end.2018.0789

16. Moses RA, Ghali FM, Pais VM, Hyams ES (2016) Unplanned Hospital Return for Infection following Ureteroscopy-Can We Identify Modifiable Risk Factors? J Urol 195:931-936. https://doi.org/10.1016/j.juro.2015.09.074 
17. Youssef RF, Neisius A, Goldsmith ZG, et al (2014) Clinical Outcomes After Ureteroscopic Lithotripsy in Patients Who Initially Presented with Urosepsis: Matched Pair Comparison with Elective Ureteroscopy. J Endourol 28:1439-1443. https://doi.org/10.1089/end.2014.0343

18. Whiteside SA, Razvi H, Dave S, et al (2015) The microbiome of the urinary tract - A role beyond infection. Nat Rev Urol 12:81-90. https://doi.org/10.1038/nrurol.2014.361

19. Kohada Y, Goriki A, Yukihiro K, et al (2019) The risk factors of urinary tract infection after transurethral resection of bladder tumors. World J Urol 37:2715-2719. https://doi.org/10.1007/s00345-019-02737-3

20. Chen D, Jiang C, Liang X, et al (2019) Early and rapid prediction of postoperative infections following percutaneous nephrolithotomy in patients with complex kidney stones. BJU Int 123:1041-1047. https://doi.org/10.1111/bju.14484

21. Fan J, Wan S, Liu L, et al (2017) Predictors for uroseptic shock in patients who undergo minimally invasive percutaneous nephrolithotomy. Urolithiasis 45:573-578. https://doi.org/10.1007/s00240-0170963-4.

Table-1: Perioperative and demographic features

\begin{tabular}{llll}
\hline & Postoperative UTI & Postoperative UTI & $\mathrm{p}$ \\
\hline & Yes (n=20) & No $(\mathrm{n}=269)$ & \\
Age (years) Ave & $47.8 \pm 12.6$ & $47.9 \pm 14.7$ & $0,988^{++}$ \\
Sex Male Female & $9(\% 45) 11(\% 55)$ & $162(\% 60.2) 107(\% 39.8)$ & $0.181^{+}$ \\
BMI Med & $26.3 \pm 3.2$ & $27.4 \pm 4.7$ & $0.436^{\S}$ \\
DM Yes No & $3(\% 15) 17(\% 85)$ & $57(\% 21.1) 212(\% 78.9)$ & $0.510^{+}$ \\
HT Yes No & $4(\% 20) 16(\% 80)$ & $69(\% 25.6) 200(\% 74.4)$ & $0.575^{+}$ \\
Charlson Comorbidity & $14(\% 70) 6(\% 30)$ & $202(\% 77.9) 57(\% 22.1)$ & $0.410^{+}$ \\
Index 0-1 [?]2 & & & \\
Stone Diameter(mm) & $13.5 \pm 4.1$ & $15 \pm 3.6$ & $0.285^{\S}$ \\
$\begin{array}{l}\text { Med } \\
\text { Stone Localisation Upper }\end{array}$ & $3(\% 15) 2(\% 10) 4(\% 20)$ & $9(\% 3.3) 29(\% 10.8) 90$ & $0.090^{+}$ \\
Calyx Middle Calyx & $8(\% 40) 0(\% 0) 3(\% 15)$ & $(\% 33.4) 72(\% 26.8) 12$ & \\
Lower Calyx Renal Pelvis & & $(\% 4.5) 67(\% 24.9)$ & \\
$\begin{array}{l}\text { Proximal Ureter Multipl } \\
\text { Preoperative }\end{array}$ & $8(\% 40) 12(\% 60)$ & $140(\% 52.1) 128(\% 47.9)$ & $0.550^{+}$ \\
$\begin{array}{l}\text { Hydronephrosis Yes No } \\
\text { UTI History Yes No }\end{array}$ & $11(\% 55) 9(\% 45)$ & $55(\% 20.5) 214(\% 79.5)$ & $\mathbf{0 . 0 0 0}{ }^{+}$ \\
$\begin{array}{l}\text { Preoperative Dj Stent } \\
\text { Yes No }\end{array}$ & $8(\% 40) 12(\% 60)$ & $63(\% 23.4) 206(\% 76.6)$ & $0.097^{+}$ \\
Access Sheat Use Yes No & $10(\% 50) 10(\% 50)$ & $157(\% 58.4) 112(\% 41.6)$ & $0.465^{+}$ \\
$\begin{array}{l}\text { Operation time (min) } \\
\text { Med }\end{array}$ & $62.5 \pm 16.6$ & $60 \pm 19.4$ & $\mathbf{0 . 0 0 8}{ }^{\S}$ \\
Residual fragment Yes No & $3(\% 30) 17(\% 70)$ & $93(\% 34.6) 176(\% 65.4)$ & $0.073^{+}$ \\
\hline
\end{tabular}

UTI: Urinary tract infection, Ave $=$ Average, $\mathrm{Med}=$ Median, $\mathrm{BMI}=$ Body mass index, $\mathrm{DM}=$ Diabetes Mellitus, $\mathrm{HT}=$ Hypertension, \pm Standard Deviation,${ }^{+}=$Chi Square Test, ${ }^{\mathrm{SS}}=$ Mann-Whitney u Test,${ }^{++}=$Independent samples t-Test

Table-2: Relationship between preoperative urine analysis and postoperative UTI

\begin{tabular}{llll}
\hline & Postoperative UTI & Postoperative UTI & p \\
\hline $\begin{array}{l}\text { Preoperative Urine } \\
\text { Analysis }\end{array}$ & Yes & No & \\
& & &
\end{tabular}




\begin{tabular}{llll}
\hline & Postoperative UTI & Postoperative UTI & $\mathrm{p}$ \\
\hline WBC Med & $22.5 \pm 196.2$ & $7.0 \pm 86.9$ & $\mathbf{0 . 0 0 9}^{\S}$ \\
Pyuria No Yes & $3(\% 15) 17(\% 85)$ & $108(\% 40.2) 161(\% 59.8)$ & $\mathbf{0 . 0 2 6}^{+}$ \\
Leukocyte esterase - + & $2(\% 10) 18(\% 90)$ & $98(\% 36.4) 171(\% 63.6)$ & $\mathbf{0 . 0 1 7}^{+}$ \\
Nitrite -+ & $13(\% 65) 7(\% 35)$ & $246(\% 91.4) 23(\% 8.6)$ & $\mathbf{0 . 0 0 0}^{+}$ \\
\hline
\end{tabular}

UTI: Urinary tract infection, Med $=$ Median, $\mathrm{WBC}=$ White blood cells, Pyuria $=\mathrm{WBC}[?] 5$ in urine

\pm Standard Deviation

${ }^{+}=$Chi Square Test, ${ }^{\mathrm{SS}}=$ Mann-Whitney u Test

Table-3 : Urine Analysis as a predictor of postoperative UTI

\begin{tabular}{lll}
\hline & Preoperative Urine Analysis for prediction of postoperative UTI & Preoperative Urine A \\
\hline & WBC + & LE + \\
Sensitivity & $85 \%$ & $90 \%$ \\
Specificity & $40.1 \%$ & $36.4 \%$ \\
PPV & $9.5 \%$ & $9.5 \%$ \\
NPV & $97.3 \%$ & $98 \%$ \\
The diagnostic odds ratio (DOR) & 3.80 & 5.16 \\
\hline
\end{tabular}

WBC: White Blood cells in urine analysis, Pyuria= WBC[?] 5 in urine

LE: Leukocyte esterase, N: Nitrite, UTI: Urinary tract infection , PPV: Positive predictive value, NPV:

Negative predictive value

Table-4: Factors associated with postoperative UTI - Univariate ve Multivariate Analysis

\begin{tabular}{llllll}
\hline & Univariate Analysis & Univariate Analysis & Univariate Analysis & Multivariate Analysis & 1 \\
\hline & OR & $95 \%$ CI & $\mathrm{p}$ & OR & \\
Preoperative Dj Stent & 2.18 & $0.85-5.57$ & 0.103 & & \\
UTI History & 4.76 & $1.87-12.04$ & 0.001 & & \\
Preoperative Hydronephrosis & 0.60 & $0.24-1.51$ & 0.280 & & \\
Access Sheat Use & 0.71 & $0.28-1.77$ & 0.467 & & \\
Operation time & 1.03 & $1.01-1.05$ & 0.025 & \\
Residual fragment & 0.34 & $0.09-1.17$ & 0.086 & & \\
WBC in urinanalysis & 1.004 & $1.001-1.007$ & 0.002 & & \\
Pyuria & 3.80 & $1.08-13.29$ & 0.036 & & \\
Leukocyte esterase positivity & 5.16 & $1.17-22.70$ & 0.030 & & \\
Nitrit positivity & 5.76 & $2.09-15.86$ & 0.001 & & \\
\hline
\end{tabular}

Cox Regression Analysis

UTI: Urinary tract infection, Pyuria $=\mathrm{WBC}[?] 5$ in urine analysis 